\title{
A Dynamic Model of Running with a Half-Circular Compliant Leg
}

\author{
M. M. ANKARALI \\ Dept. of Mechanical Engineering, \\ Johns Hopkins University, Baltimore 21218, MD, USA \\ E-mail: mertankarali@jhu.edu \\ E. SAYGINER* ${ }^{*}$ Y. YAZICIOGLU ${ }^{\S}$, A. SARANLI ${ }^{\ddagger}$ and U. SARANLI ${ }^{\dagger}$ \\ *Dept. of Information Systems, ${ }^{\S}$ Dept. of Mechanical Engineering, \\ ${ }^{\ddagger}$ Dept. of Electrical and Electronics Eng. ${ }^{\dagger}$ Dept. of Computer Engineering, \\ Middle East Technical University, Ankara 06800, Turkey \\ E-mail: *sayginer@metu.edu.tr, ${ }^{\S} y i g i t @ m e t u . e d u . t r,{ }^{\ddagger}$ afsars@metu.edu.tr, \\ $\dagger$ †saranli@ceng.metu.edu.tr
}

\begin{abstract}
Substantial research on legged robotics focuses on the design and morphology of leg structures. In this context, the advantages of passive compliance for reliability and simplicity have long been recognized. For example, composite leg designs with a half-circular profile used on later versions of the RHex hexapod were found to support a rich set of dynamic behaviors. However, the complex geometry and compliance properties of these legs have been difficult to model, preventing the use of dynamic simulations. In this paper, we present a simple dynamic model for this leg design for a planar monopod, taking into account both the kinematics of rolling contact and the nonlinear compliance of the geometry. We show through simulations that realistic predictions for system trajectories can be generated and leave experimental validation of this model for future work.
\end{abstract}

Keywords: RHex, Half-Circular Compliant Leg, Castigliano's Theorem

\section{Introduction}

Legged morphologies admit a wide range of behavioral alternatives through behaviors such as running, self-righting and climbing. The RHex hexapod ${ }^{1}$ has been a good example of how biological inspiration can help improve mobility, motivating more recent instantiations of similar ideas. ${ }^{2} \mathrm{~A}$ distinguishing feature of recent versions of RHex are its half-circular, compliant leg designs that were found to have robust mechanical properties and good 
dynamic performance but have been difficult to model accurately. Consequently, existing behavioral simulations to study this robot were mostly based on a radially compliant, telescoping leg structure, ${ }^{3,4}$ closer to what has been used on monopedal or bipedal robot designs. In this paper, we present a new model that incorporates both the kinematics of rolling contact and the nonlinear compliance associated with RHex's composite halfcircular legs, extending on previous modeling attempts towards a uniform model to support complete dynamic simulations of the robot. ${ }^{5}$ An alternative approach to solve this modeling problem is through the use of numerical simulations based on finite-element methods. ${ }^{6}$ However such methods are not only computationally demanding, but they also do not provide any insight into the underlying dynamics of the compliant mechanism to support the analysis and control of the overall system.

Some of the components for the model we present in this paper were introduced by earlier work. ${ }^{7}$ However, this paper provides a more detailed kinematic model of the leg as well as additional novel components such as the formulation of the no-slip constraint for ground contact and hybrid transitions for running dynamics. For clarity, we present our leg model in the context of a lossless monopedal runner, the C-Pod, which has simple but sufficiently rich dynamics to illustrate basic properties of our model. The morphology of this system is similar to the TD-SLIP model from our earlier work, ${ }^{8}$ incorporating both damping and hip actuation but using the a compliant half-circular leg morphology instead of a telescoping design.

\section{Phases, Constraints and Kinematics of C-Pod}
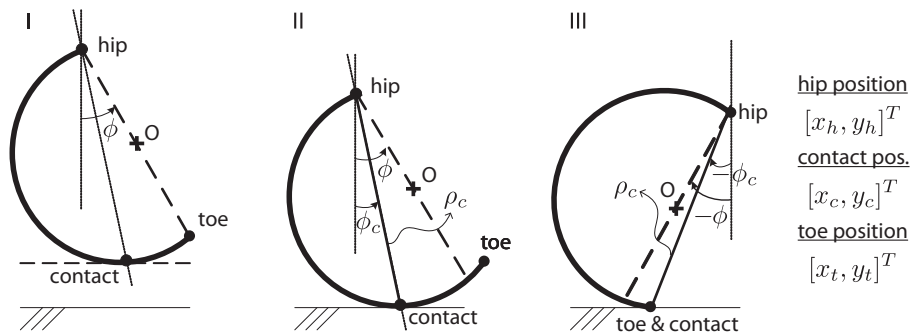

Fig. 1. Phases for C-Pod running: I - flight, II - rolling stance and III - toe stance. Point O marks the center of the leg circle. Dashed lines show the diameter of the arc passing through the hip, whose angle with the vertical defines the leg angle $\phi$.

Similar to all legged systems, the C-Pod is a hybrid dynamical system. 
As illustrated in Fig. 1, the model goes through three phases during running: I. Flight, II. rolling stance and III. toe stance. During flight, the leg is assumed to be massless and the body behaves as a point-mass acted upon by gravity. Unlike other commonly used monopedal models, ${ }^{8}$ two different stance phases are necessary for C-Pod to capture the effects of the limited arc length of the circular leg. The dynamics associated with these two phases are the primary focus of this paper. The vector $\left[x_{c}, y_{c}\right]$ denotes the point on the leg closest to the ground, with $y_{c}=0$ whenever the system is in stance. During stance, the rolling phase during stance is in effect whenever $x_{c} \neq$ $x_{t}$, with ground contact falling on the circular part of the leg. Otherwise, the contact point is at the toe and the system is in its toe stance phase. The absolute horizontal location of the leg and the kinematics of rolling during stance are determined when the leg touches the ground based on the associated arc-length $l_{t d}$ and the contact position $x_{c, t d}$, where $l$ denotes the length of the arc between the contact and hip position and sub-script "td" stands for touchdown.

Our half-circular leg model is based on two basic assumptions:

(1) The leg always maintains a circular shape parametrized through its radius but preserves its total arc length,

(2) The leg undergoes pure rolling motion during stance with no slippage, corresponding to the constraint

$$
l-l_{t d}=x_{c}-x_{c, t d}
$$

to be maintained throughout the rolling stance phase.

The realization of these assumptions is one of the main contributions of this paper, allowing us to formulate system kinematics during rolling stance based only on the coordinates of the hip joint and the touchdown parameters $l_{t d}$ and $x_{c, t d}$. Kinematically, the leg angle $\phi$ can be found during stance through the solution to

$$
y_{h}(\sin \phi-\pi+\phi)+(1+\cos \phi)\left(x_{h}-x_{c, t d}+l_{t d}\right)=0,
$$

based on which all other kinematic stance variables can also be found as detailed in Table 1.

Since C-Pod is a hybrid dynamical system, kinematic boundaries must be defined for phase transitions. Transition from flight to stance happens when the fully extended circular leg comes into contact with the ground. In contrast, two transitions are possible during the rolling phase. If the ground reaction force becomes zero, the leg lifts-off and the system transitions into 
Table 1. Kinematic solutions during the rolling stance phase

\begin{tabular}{|c|l|l|}
\hline Quantity & Derivation & Description \\
\hline \hline$\phi_{c}$ & $=\phi / 2$ & Angle of the "virtual spring" \\
\hline$r$ & $=y_{h} /(1+\cos \phi)$ & Leg angle and radius \\
\hline$\rho_{c}$ & $=2 r \cos \phi$ & Angle of the "virtual spring" \\
\hline$l$ & $=(\pi-\phi) r$ & Arc length of the compressed section \\
\hline$x_{c}$ & $=l-l_{t d}+x_{c, t d}$ & Horizontal contact position \\
\hline
\end{tabular}

flight. If, however, the arc-length of the compressed section becomes equal to the arc-length of the leg, the system transitions into toe stance.

During toe stance, the leg acts as a nonlinear radial spring with a fixed toe. Since we know the contact position, the length and angle of this "virtual spring" can be computed as

$$
\rho_{c}=\left\|\mathbf{v}_{c}\right\|_{2}, \quad \phi_{c}=\operatorname{atan} 2\left(\mathbf{v}_{c}(1),-\mathbf{v}_{c}(2)\right),
$$

where $\mathbf{v}_{c}=\left[x_{c}, y_{c}\right]^{T}-\left[x_{h}, y_{h}\right]^{T}$ is defined as the virtual leg vector. The hip angle $\phi$ during toe-stance is then found by numerically solving

$$
\pi+2\left(\phi_{c}-\phi\right)-2 l_{0} \cos \left(\phi_{c}-\phi\right) / \rho_{c}=0,
$$

where $l_{0}$ is the arc length of the whole leg. Remaining kinematic parameters during toe-stance are found using direct algebraic relations.

\section{Stance Dynamics of the Half-Circular Compliant Leg}

The dynamics of the C-Pod system during stance are governed by the force generated by the compressed half-circular leg, which we approximate using an adaptation of Castiglianos theorem. ${ }^{9}$ In the absence of a hip torque and with a massless leg, the leg force must act along $\mathbf{v}_{c}$ (i.e. along the line connecting the hip and contact point). Our method relies on finding the total strain energy in the compliant leg as a function of the ground reaction force, whose derivative with respect to the force yields the instantaneous deflection of the leg. This can then be used to compute the instantaneous stiffness as

$$
k(r, l)=\frac{2 E I / r^{3}}{-3 \sin (l / r)+(\cos (l / r)+2)(l / r)},
$$

which only depends on the radius and arc length of the compressed leg segment, making it applicable to both stance phases. Consistent with our assumption above, we consider the rest leg length $\rho_{c, 0}$ to be the distance between the current contact point and the hip for an uncompressed leg, 
which can be computed using

$$
\rho_{c, 0}=2 r_{0} \sin \left(\frac{l}{2 r_{0}}\right)
$$

where $r_{0}$ is the radius of the uncompressed leg. The definition of (3) is structurally different than previously proposed definitions, ${ }^{7}$ which were prone to unrealistic discontinuities in leg forces during hybrid transitions between rolling and toe stance phases. Our model ensures continuity of leg forces during such transitions.

Subsequently, we use the deflection $\delta=\rho_{c, 0}-\rho_{c}$ to compute the leg force as $F=k(r, l) \delta$. Finally, this leg force and the fact that the leg force acts along $\mathbf{v}_{c}$ can be used to finalize the dynamics of the C-Pod body as

$$
\left[\begin{array}{c}
\ddot{x}_{h} \\
\ddot{y}_{h}
\end{array}\right]=-\frac{\mathbf{v}_{c}}{\rho_{c}} F / m+\left[\begin{array}{c}
0 \\
-g
\end{array}\right],
$$

where $m$ and $g$ corresponds to the body mass and gravity, respectively.

\section{Simulation Results}
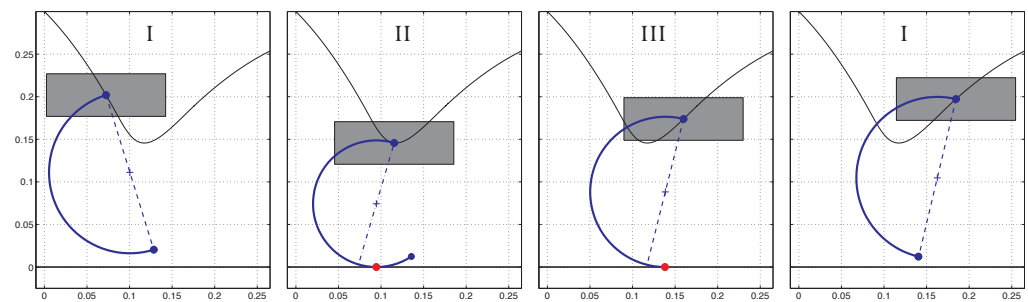

Fig. 2. Snapshots from an example simulation within a single stride. Black curve is the the body trajectory for the whole stride.

Fig. 2 illustrates four snapshots of a simulated C-Pod from a single stride, with the system phase indicated in roman numerals for each snapshot. Our results show that the trajectory and the evolution of all system variables are qualitatively realistic, noting that an experimental validation of these results is still necessary to reach any quantitative conclusions about their accuracy. In the absence of such a validation, which we intend to perform in the near future on a recently completed monopedal platform, the only qualitative evaluation we can perform is an evaluation of whether the compliance model achieves conservation of energy for a lossless system structure. 
In this context, we simulated a single stride of the C-Pod model using a range of different initial conditions and we observed relative energy differences after a full stride. Simulations were done with $m=1.5 \mathrm{~kg}$ (mass per each leg for RHex), $r_{0}=0.19 m, E I=2.7859 \mathrm{~N}$ ( $\mathrm{E}$ and I are the modulus of elasticity and moment of area of the leg cross-section, respectively), selected to closely match physical properties of our RHex robot. The range of initial conditions we considered were $y_{h} \in\left[r_{0}, 2 r_{0}\right] m, \dot{x}_{h} \in\left[-10 r_{0}, 10 r_{0}\right] \mathrm{m} / \mathrm{s}$ and $\phi_{t d} \in\left[-45^{\circ}, 45^{\circ}\right]$. For each simulation, we evaluated the percentage energy change in a single stride, defined as

$$
P E:=100 \frac{\left|E_{k}-E_{k+1}\right|}{E_{k}}
$$

Table 2 summarizes our results. We observed that the mean energy difference after a full stride across all simulations was approximately $2 \%$, which is reasonable given that energy losses will be on the order of $\% 30-\% 50$ for physical robotic systems with non-negligible damping. ${ }^{10,11}$ Interestingly, energy changes for strides without the rolling phase (i.e. only toe-stance) were negligible, showing that our rest length and non-linear stiffness definitions provide a fully conservative spring model. Consequently, small changes in energy occur mainly due to the rolling phase, which is not unexpected since the kinematics during the rolling phase inherently depend on our first assumption of the leg preserving its circular shape, potentiall resulting in approximation errors.

Table 2. Apex to apex percentage energy changes for a single stride with the C-Pod model.

\begin{tabular}{|l|c|c|}
\hline & $\mu \pm \sigma$ for $P E$ & $P E_{\max }$ \\
\hline \hline All strides considered & $1.88 \pm 1.66$ & 6.03 \\
\hline Strides with only rolling stance & $2.16 \pm 1.48$ & 5.92 \\
\hline Strides with only toe-stance & $0.00 \pm 0.00$ & 0.00 \\
\hline Strides with both stance phases & $2.35 \pm 1.59$ & 6.03 \\
\hline
\end{tabular}

In addition to the results presented in Table 2, we illustrate single stride energy changes as a function of initial height in Fig. 3. In a somewhat expected manner, an increase in initial height results in an associated increase in the energy errors. This is mainly because the amount of leg compression also increases with larger initial apex heights, making the impact of our approximations and assumptions more significant. Most mathematical models for compliant systems assume small deflections ${ }^{12}$ and Castigliano's theorem underlying our model is no exception. For these reasons, the positive correlation between initial apex height and energy errors we observed in our 
simulations are reasonable and expected. Fortunately, the maximum height that RHex can reach even for its fastest speeds is well below $2 r_{0}$, suggesting that energy errors exhibited by our model should not be unreasonable under normal operating conditions.

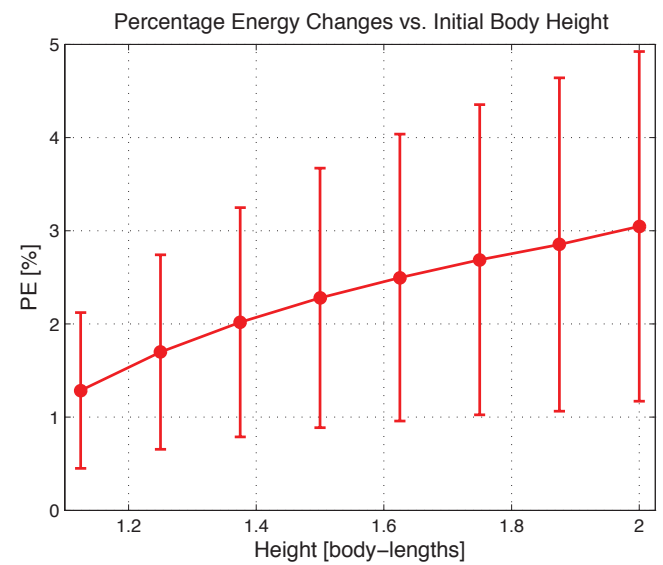

Fig. 3. Percentage energy changes as a fucntion of initial apex height. The vertical bars and solid markers represent the corresponding standard deviation and mean.

\section{Conclusion}

In this papaer, we proposed a dynamic model of running with a half-circular compliant leg, which is one of the core properties of the recent versions of the RHex robots. We had taken into account both the complex kinematics of rolling contact under the assumption of no-slippage over the ground and the nonlinear compliance of the geometry. We show through simulations that realistic predictions for system trajectories can be generated. We also observed that energy differences of the lossless and non-actuated C-Pod are negligible compared the energy losses observed in real robots. However in order to test the accuracy and reliability of our model, we have to perform an experimental validation that we leave as our future work.

\section{Acknowledgments}

We would like to thank Yasemin O. Aydin for her support in our comparisons with alternate models. This project was partially supported by TUBITAK project 109E032. 


\section{References}

1. U. Saranli, M. Buehler and D. E. Koditschek, RHex: A simple and highly mobile robot, International Journal of Robotics Research 20, 616(July 2001).

2. D. Goldman, H. Komsuoglu and D. E. Koditschek, March of the sandbots, IEEE Spectrum 46, 30(April 2009).

3. M. Ankarali and U. Saranli, Control of underactuated planar pronking through an embedded spring-mass hopper template, Autonomous Robots 30, 217 (2011).

4. U. Saranli, Dynamic locomotion with a hexapod robot, $\mathrm{PhD}$ thesis, University of Michigan, (Ann Arbor, MI, USA, 2002).

5. Y. O. Aydin, K. C. Galloway, Y. Yazicioglu and D. E. Koditschek, Modeling the compliance of a variable stiffness C-shaped leg using Castigliano's theorem, in ASME Conference Proceedings, (44106)2010.

6. G. Oral, Flexible multibody dynamic modelling and simulation of RHex hexapod robot with half circular compliant legs, $\mathrm{PhD}$ thesis, Middle East Technical University, (Ankara, Turkey, 2008).

7. E. Sayginer, Modelling the effects of half circular compliant legs on the kinematics and dynamics of a legged robot, PhD thesis, Middle East Technical University, (Ankara, Turkey, 2010).

8. M. M. Ankarali and U. Saranli, Stride-to-stride energy regulation for robust self-stability of a torque-actuated dissipative spring-mass hopper, Chaos 20(Sep. 2010).

9. J. E. Shigley and C. R. Mischke, Mechanical Engineering Design (McGrawHill, 1989).

10. M. Ahmadi and M. Buehler, The ARL monopod II running robot: Control and energetics, in Proceedings of the International Conference on Robotics and Automation, 1999.

11. J. Hurst and A. Rizzi, Series compliance for an efficient running gait, IEEE Robotics Automation Magazine 15, 42 (september 2008).

12. U. Saranli, O. Arslan, M. M. Ankarali and O. Morgul, Approximate analytic solutions to non-symmetric stance trajectories of the passive spring-loaded inverted pendulum with damping, Nonlinear Dynamics 62, 729 (2010). 